\title{
85274 - ADAPTAÇÃO TRANSCULTURAL E VALIDAÇÃO DO THE STROKE SELF-EFFICACY QUESTIONNAIRE PARA O PORTUGUÊS DO BRASIL: UM ESTUDO PILOTO
}

\author{
Pôster - Gerontologia
}

Eliana da Silva Jaques / Jaques, ES / PUCRS; Pedro Henrique Deon / Deon, PH / PUCRS; Fabiane de Oliveira Brauner / Brauner, FO / PUCRS; Bianca Pacheco Loss / Loss, BP / PUCRS; Gabriel Hoff da Silveira / da Silveira, GH / PUCRS; Régis Gemerasca Mestriner / Mestriner, RG / PUCRS

Introdução: $\mathrm{O}$ acidente vascular cerebral (AVC) é uma doença caracterizada pelo comprometimento funcional do cérebro, podendo ser dividido em AVC isquêmico e hemorrágico. As doenças cerebrovasculares podem gerar consequências, entre elas o déficit nas funções motoras, sensoriais, cognitivas, comunicativas e emocionais. A paralisia/paresia é o déficit mais comum relacionado às funções motoras e ocorre, tipicamente, na região contralateral a área cerebral afetada. Assim, os pacientes podem ser afetados por transtornos emocionais, além de prejudicar a sua capacidade de realizar as suas atividades básicas e instrumentais de vida diária. A auto percepção funcional é um importante mediador do processo de adaptação pós-AVC. Porém, poucos estudos buscam mensurar as restrições de participação percebidas pelo indivíduo em seu processo de reabilitação. O The Stroke Self-Efficacy Questionnaire (SSEQ) é um excelente instrumento para avaliar o grau de confiança do indivíduo frente à diversas atividades de sua rotina diária, e ainda não foi validado transculturalmente para a língua portuguesa, impossibilitando que os profissionais do nosso país possam utilizá-lo para melhor traçar os objetivos da reabilitação. Objetivo: Adaptar transculturalmente, examinar a consistência interna e a convergência de desempenho de uma versão brasileira do SSEQ. Material e métodos: Tradução e adaptação transcultural por meio do método de Beaton. A consistência interna foi aferida por meio do alfa de Cronbach e a avaliação convergente comparando a SSEQ - versão brasileira com os resultados dos Índices de Barthel, Franchay e Questionário de Qualidade de vida pós-AVC (QoL pós-AVC). Resultados: Neste estudo, 12 indivíduos foram avaliados. O SSEQ - versão brasileira atingiu uma consistência interna substancial, com um alfa de Cronbach de 0,754. Observou-se também uma boa validade convergente quando comparado com outros instrumentos padrão áureo, sendo mais consistente com os índices de Barthel (ICC = 0,77) e Frenchay (ICC $=0,63$ ), respectivamente. Não existiu concordância estatisticamente significativa em comparação com o QoL pós-AVC $(\mathrm{ICC}=0,41)$. Conclusão: Conclui-se, preliminarmente, que o SSEQ - versão brasileira possui boas características psicométricas e é consistente com os resultados aferidos por outros instrumentos padrão-áureo. Pretende-se ampliar a amostra para a validação final do instrumento em questão.

Palavras-chave: Acidente vascular cerebral; Estudo de validação; Reabilitação. 